\title{
ON THE ŁOJASIEWICZ EXPONENT FOR ANALYTIC CURVES
}

\author{
JACEK CHA̧DZYŃSKI and TADEUSZ KRASIŃSKI \\ Faculty of Mathematics, University of Łódź \\ S. Banacha 22, 90-238 Eódź, Poland \\ E-mail: jachadzy@imul.uni.lodz.pl,krasinsk@krysia.uni.lodz.pl
}

\author{
Dedicated to Professor Stanistaw Eojasiewicz
} on the occasion of his 70th birthday

\footnotetext{
Abstract. An effective formula for the Łojasiewicz exponent for analytic curves in a neighbourhood of $0 \in \mathbb{C}^{m}$ is given.

1. The Łojasiewicz exponent for sets. In this section we shall assume that $\Omega$ is a neighbourhood of $0 \in \mathbb{C}^{m}(m \geq 2), X, Y$ - analytic sets in $\Omega$ and $X \cap Y=\{0\}$.

Let

$$
\begin{array}{r}
N(X, Y)=\left\{\nu \in \mathbb{R}_{+}: \exists A>0, \exists B>0, \forall z \in \Omega,|z|<B\right. \\
\left.\quad \Rightarrow \varrho(z, X)+\varrho(z, Y) \geq A|z|^{\nu}\right\},
\end{array}
$$
}

here $|\cdot|$ is the polycylindric norm and $\varrho(\cdot, Z)$ is the distance function to a set $Z$. One can prove (see $\left[\mathrm{E}_{1}\right], \mathrm{IV} .7$ ) that under the above assumption $N(X, Y)$ is not empty.

By the Eojasiewicz exponent of $X, Y$ at 0 we mean $\inf N(X, Y)$ and denote it by $\mathcal{L}_{0}(X, Y)$.

One can prove

Proposition 1 ([ $\left[\mathrm{E}_{2}\right]$, s. 18). If $X, Y$ satisfy the above assumptions and 0 is an accumulation point of $X$, then

$$
N(X, Y)=\left\{\nu \in \mathbb{R}_{+}: \exists A>0, \exists B>0, \forall x \in X,|x|<B \Rightarrow \varrho(x, Y) \geq A|x|^{\nu}\right\} .
$$

This research was realized within the project No.2P03A05010 financed in 1996-1998 by KBN. 1991 Mathematics Subject Classification: Primary 32S05; secondary 32B10.

Key words: Łojasiewicz exponent, analytic curve.

Received by the editors: September 27, 1996.

The paper is in final form and no version of it will be published elsewhere. 
Proposition 2 ([T], Thm. 3.2). If $X, Y$ satisfy the same assumptions as above, then $\mathcal{L}_{0}(X, Y) \in N(X, Y)$.

Estimations of $\mathcal{L}_{0}(X, Y)$ from above are known. P. Tworzewski and E. Cygan in [T] and $[\mathrm{CT}]$ gave such estimations in terms of the intersection multiplicity of $X$ and $Y$ in both cases: 0 is or not an isolated point of $X \cap Y$.

Let us note an easy property of $\mathcal{L}_{0}(X, Y)$. Let $X=X_{1} \cup \ldots \cup X_{r}, Y=Y_{1} \cup \ldots \cup Y_{s}$, where $X_{1}, \ldots, X_{r}, Y_{1}, \ldots, Y_{s}$ are analytic sets in $\Omega$ passing through $0 \in \mathbb{C}^{m}$.

Proposition 3. Under the above assumptions

$$
\mathcal{L}_{0}(X, Y)=\max _{k, l} \mathcal{L}_{0}\left(X_{k}, Y_{l}\right) .
$$

2. The Łojasiewicz exponent for mappings. Let $\Omega \subset \mathbb{C}^{n}(n \geq 2)$ be a neighbourhood of the origin, $F=\left(f_{1}, \ldots, f_{m}\right): \Omega \rightarrow \mathbb{C}^{m}$ be a holomorphic mapping having an isolated zero at $0 \in \mathbb{C}^{n}$. Let $S$ be an analytic set in $\Omega$ such that 0 is an accumulation point of $S$. Put

$$
N(F \mid S)=\left\{\nu \in \mathbb{R}_{+}: \exists A>0, \exists B>0, \forall z \in S,|z|<B \Rightarrow A|z|^{\nu} \leq|F(z)|\right\} .
$$

When $S=\Omega$ we define $N(F)=N(F \mid \Omega)$.

By the Eojasiewicz exponent of $F \mid S$ at 0 we mean $\mathcal{L}_{0}(F \mid S)=\inf N(F \mid S)$. Analogously, $\mathcal{L}_{0}(F)=\inf N(F)$.

In the sequel for a holomorphic function $g: \Omega \rightarrow \mathbb{C}$ we put $V(g):=\{z \in \Omega: g(z)=0\}$.

One can prove

TheOREM 1 ([CK]). If $\Omega \subset \mathbb{C}^{n}(n \geq 2)$ is a neighbourhood of the origin, $F=$ $\left(f_{1}, \ldots, f_{m}\right): \Omega \rightarrow \mathbb{C}^{m}$ is a holomorphic mapping having an isolated zero at $0 \in \mathbb{C}^{n}$ and $f:=f_{1} \cdot \ldots \cdot f_{m}$, then

$$
\mathcal{L}_{0}(F)=\mathcal{L}_{0}(F \mid V(f)) .
$$

We shall now prove a theorem on the Eojasiewicz exponent, needed in the sequel.

Let $n=2$ and $\Omega$ be a neighbourhood of $0 \in \mathbb{C}^{2}, F=\left(f_{1}, \ldots, f_{m}\right): \Omega \rightarrow \mathbb{C}^{m}$ be a holomorphic mapping having an isolated zero at $0 \in \mathbb{C}^{2}$.

THEOREM 2. If $f_{1}$ is a homogeneous form of degree $r$ with $r$ different tangent lines and $r \leq$ ord $f_{i}<\infty$, then

$$
\mathcal{L}_{0}(F)=\mathcal{L}_{0}\left(F \mid V\left(f_{1}\right)\right) .
$$

Proof. Let $f_{1}=L_{1} \cdot \ldots \cdot L_{r}$ be a factorization of $f_{1}$ into linear factors. Let $\mu(g, h)$ denote the multiplicity of a mapping $(g, h): \Omega \rightarrow \mathbb{C}^{2}$ at $0 \in \mathbb{C}^{2}$. Since

$$
\mathcal{L}_{0}\left(F \mid V\left(f_{1}\right)\right)=\max _{i=1}^{r} \mathcal{L}_{0}\left(F \mid V\left(L_{i}\right)\right)=\max _{i=1}^{r} \min _{j=2}^{m} \mu\left(L_{i}, f_{j}\right),
$$

then, without loss of generality, we may assume that

$$
\mathcal{L}_{0}\left(F \mid V\left(f_{1}\right)\right)=\mu\left(L_{1}, f_{m}\right) .
$$

Hence for each $i \in\{1, \ldots, r\}$ there exists $j \in\{1, \ldots, m\}$ such that

$$
\mu\left(L_{i}, f_{j}\right) \leq \mu\left(L_{1}, f_{m}\right) .
$$


By Theorem 1 we have

$$
\mathcal{L}_{0}(F)=\mathcal{L}_{0}(F \mid V(f)) .
$$

Let $\mathcal{O}^{2}$ be the ring of germs of holomorphic functions at $0 \in \mathbb{C}^{2}, h: \Omega \rightarrow \mathbb{C}-$ a holomorphic function and $\hat{h} \in \mathcal{O}^{2}$ - the germ generated by $h$. Assume that $\hat{h}$ is an arbitrary irreducible germ dividing $\hat{f}$. It is easy to check that

$$
\mathcal{L}_{0}(F \mid V(f))=\max _{h} \mathcal{L}_{0}(F \mid V(h)) .
$$

It follows from (1), (3) and (4) that it suffices to show that

$$
\mathcal{L}_{0}(F \mid V(h)) \leq \mu\left(L_{1}, f_{m}\right) .
$$

Assume to the contrary that (5) does not hold for some $h$. In the sequel ord $h$ means the order of $h$ at $0 \in \mathbb{C}^{2}$. Since

$$
\mathcal{L}_{0}(F \mid V(h))=(1 / \text { ord } h) \min _{k=1}^{m} \mu\left(f_{k}, h\right),
$$

then for every $k \in\{1, \ldots, m\}$ we have

$$
\mu\left(L_{1}, f_{m}\right)<\mu\left(f_{k}, h\right) / \text { ord } h .
$$

If the curve $V(h)$ has no common tangent line with the curve $V\left(f_{1}\right)$ at 0 , then

$$
\mu\left(f_{1}, h\right) / \text { ord } h=r \leq \mu\left(L_{1}, f_{m}\right)
$$

which contradicts $(6)$.

So, assume that the line $L_{i}=0$ is tangent to $V(h)$ at 0 . Then, there exists $j \in$ $\{1, \ldots, m\}$ such that (2) holds. If $L_{i}=0$ is not tangent to $V\left(f_{j}\right)$ at 0 , then

$$
\mu\left(h, f_{j}\right) / \operatorname{ord} h=\operatorname{ord} f_{j}=\mu\left(L_{i}, f_{j}\right) \leq \mu\left(L_{1}, f_{m}\right),
$$

which contradicts (6). If $L_{i}=0$ is tangent to $V\left(f_{j}\right)$ at 0 , we put $s:=\mu\left(L_{i}, f_{j}\right)$. Then we have $r \leq r_{j}:=$ ord $f_{j}<s$. Since the considerations are local, then shrinking $\Omega$, if necessary, we may assume that $f_{j}=\sum_{\nu=r_{j}}^{\infty} P_{\nu}$, where $P_{\nu}$ is a homogeneous polynomial of degree $\nu$. Let $f_{j}^{*}:=\sum_{\nu=s}^{\infty} P_{\nu}$. Take arbitrary $\nu \in\left\{r_{j}, \ldots, s-1\right\}$. Then from the assumption that $f_{1}$ has $r$ different tangent lines we have

$$
\mu\left(P_{\nu}, h\right) \geq \mu\left(L_{i}, h\right)+(\nu-1) \text { ord } h \geq \mu\left(L_{i}, h\right)+(r-1) \text { ord } h=\mu\left(f_{1}, h\right) .
$$

Hence

$$
\mu\left(f_{j}-f_{j}^{*}, h\right) \geq \mu\left(f_{1}, h\right) .
$$

On the other hand, from (2) and (6) for $k=1$ we have

$$
\mu\left(f_{j}^{*}, h\right)=s \text { ord } h=\mu\left(L_{i}, f_{j}\right) \text { ord } h \leq \mu\left(L_{1}, f_{m}\right) \text { ord } h<\mu\left(f_{1}, h\right) .
$$

Hence and from (7)

$$
\mu\left(f_{j}, h\right)=\mu\left(f_{j}^{*}, h\right) \leq \mu\left(L_{1}, f_{m}\right) \text { ord } h,
$$

which contradicts $(6)$

This ends the proof. 
3. Main results. In this section we shall give an effective formula for the Łojasiewicz exponent for analytic curves (Theorems 3 and 4).

Let, in the sequel, $\Omega$ be a neighbourhood of $0 \in \mathbb{C}^{m}(m \geq 2), X, Y$ - analytic curves in $\Omega$ (i.e. analytic sets of pure dimension 1) and $X \cap Y=\{0\}$. Since the considerations are local, we may assume that $X=X_{1} \cup \ldots \cup X_{r}, Y=Y_{1} \cup \ldots \cup Y_{s}$, where $X_{i}, Y_{j}$ are analytic curves in $\Omega$ generating irreducible germs at 0 . Hence and from Proposition 3 it follows that the problem of finding the Łojasiewicz exponent for $X, Y$ reduces to the case when $X$ and $Y$ generate irreducible germs at 0 .

Let now $Z$ be an analytic curve in $\Omega$ generating an irreducible germ at 0 . Then $Z$ has only one tangent at 0 . Without loss of generality, changing the coordinates linearly in $\mathbb{C}^{n}$, if necessary, we may assume that this tangent does not lie in the hyperplane $H_{1}:=\left\{\left(z_{1}, \ldots, z_{m}\right) \in \mathbb{C}^{m}: z_{1}=0\right\}$. Shrinking $\Omega$, we may equivalently express this situation in terms of a holomorphic description of $Z$. Namely, by the second version of the Puiseux theorem ([ $\left.\mathrm{E}_{1}\right]$, II.6.2) we get easily

Proposition 4. A curve $Z$ generates an irreducible germ at 0 and has the tangent not lying in $H_{1}$ if and only if in a neighbourhood $\Omega^{\prime} \subset \Omega, Z$ can be represented in the form

$$
Z \cap \Omega^{\prime}=\left\{\left(t^{r}, \lambda_{2}(t), \ldots, \lambda_{m}(t)\right): t \in W\right\},
$$

where $r$ is a positive integer, $W$ - a neighbourhood of 0 in $\mathbb{C}, \lambda_{j}$ - holomorphic functions in $W$ such that ord $\lambda_{j} \geq r$ for $j=2, \ldots, m$.

If the above mapping $W \ni t \mapsto\left(t^{r}, \lambda_{2}(t), \ldots, \lambda_{m}(t)\right) \in Z \cap \Omega^{\prime}$ is a homeomorphism we shall call this mapping a parametrization of $Z \cap \Omega^{\prime}$.

Now, we shall give a formula for $\mathcal{L}_{0}(X, Y)$ in terms of holomorphic descriptions of $X$ and $Y$. The assumptions, under which the formula will be obtained, are not restrictive. It follows from both Proposition 4 and its precedent considerations.

First, we fix some standard notations. Let $\lambda=\left(\lambda_{2}, \ldots, \lambda_{m}\right), \varphi=\left(\varphi_{2}, \ldots, \varphi_{m}\right)$, $\psi=\left(\psi_{2} \ldots, \psi_{m}\right)$ be holomorphic mappings in a neighbourhood of $0 \in \mathbb{C}$. Then we define ord $\lambda:=\min _{i=2}^{m}$ ord $\lambda_{i}$ and $\varphi-\psi:=\left(\varphi_{2}-\psi_{2}, \ldots, \varphi_{m}-\psi_{m}\right)$.

Let $\Omega$ be a neighbourhood of $0 \in \mathbb{C}^{m}(m \geq 2)$ and $X, Y$ - analytic curves in $\Omega$.

Theorem 3. If $X=\left\{\left(t^{p}, \varphi(t)\right): t \in U\right\}, Y=\left\{\left(t^{q}, \psi(t)\right): t \in V\right\}$, where $p, q$ are positive integers, $U, V$ - neighbourhoods of 0 in $\mathbb{C}, \varphi, \psi$ - holomorphic mappings satisfying ord $\varphi \geq p$, ord $\psi \geq q$ and $X \cap Y=\{0\}$, then

$$
\begin{aligned}
\mathcal{L}_{0}(X, Y) & =(1 / p q) \max _{i=1}^{q} \text { ord }\left(\varphi\left(t^{q}\right)-\psi\left(\eta^{i} t^{p}\right)\right) \\
& =(1 / p q) \max _{i=1}^{p} \text { ord }\left(\psi\left(t^{p}\right)-\varphi\left(\varepsilon^{i} t^{q}\right)\right),
\end{aligned}
$$

where $\eta$, $\varepsilon$ mean primitive roots of unity of degree $q$ and $p$, respectively.

Proof. By the symmetry of $X$ and $Y$ it suffices to prove the first formula in (8). Denote by $\nu$ the right hand side of the first equality in (8). For simplicity, we may assume that

$$
\nu=(1 / p q) \operatorname{ord}\left(\varphi\left(t^{q}\right)-\psi\left(t^{p}\right)\right) .
$$


Put $d:=p q$. From the assumptions and (9) we get that there exist constants $C_{1}, D_{1}$, $D_{2}, r>0$ such that for $|t|<r$

$$
\begin{gathered}
t^{q} \in U, \quad t^{p} \in U \cap V, \\
C_{1}|t|^{d} \leq\left|\left(t^{d}, \varphi\left(t^{q}\right)\right)\right| \leq D_{1}|t|^{d}, \\
\left|\varphi\left(t^{q}\right)-\psi\left(t^{p}\right)\right| \leq D_{2}|t|^{\nu d} .
\end{gathered}
$$

Let $P(\delta):=\left\{z \in \mathbb{C}^{m}:|z|<\delta\right\}$. Take additionally $\delta>0$ such that $P(2 \delta) \subset \Omega$ and $2 \delta<r^{d}$.

Since $0 \in \mathbb{C}^{m}$ is an accumulation point of $X$, then by Proposition 1 it suffices for $x \in X \cap P(\delta)$ to estimate $\varrho(x, Y)$ from above and from below by $|x|^{\nu}$.

Let $U^{*}:=\left\{t \in \mathbb{C}: t^{q} \in U\right\}$ and $V^{*}:=\left\{t \in \mathbb{C}: t^{p} \in V\right\}$.

First, we estimate $\varrho(x, Y)$ from above for $x \in X \cap P(\delta)$. Let $x=\left(t^{d}, \varphi\left(t^{q}\right)\right)$. From the definition of infimum and (10), (11), (12) we have

$$
\begin{aligned}
\varrho(x, Y) & =\inf _{\tau \in V^{*}}\left|\left(t^{d}-\tau^{d}, \varphi\left(t^{q}\right)-\psi\left(\tau^{p}\right)\right)\right| \\
& \leq\left|\left(0, \varphi\left(t^{q}\right)-\psi\left(t^{p}\right)\right)\right| \leq D_{2}|t|^{\nu d} \leq D|x|^{\nu},
\end{aligned}
$$

where $D:=D_{2} / C_{1}^{\nu}$.

Consider the mapping $F: U^{*} \times V^{*} \ni(t, \tau) \mapsto\left(t^{d}-\tau^{d}, \varphi\left(t^{q}\right)-\psi\left(\tau^{p}\right)\right) \in \mathbb{C}^{m}$. The mapping has an isolated zero at $0 \in \mathbb{C}^{2}$. From the definition of the Łojasiewicz exponent, diminishing $r$ if necessary, we have that there exists $C_{2}>0$ such that for $|(t, \tau)|<r$

$$
|F(t, \tau)| \geq C_{2}|(t, \tau)|^{\mathcal{L}_{0}(F)}
$$

Let us calculate $\mathcal{L}_{0}(F)$. It is easy to check that $F$ satisfies the assumption of Theorem 2. Then $\mathcal{L}_{0}(F)=\mathcal{L}_{0}\left(F \mid \Gamma_{1}\right)$, where $\Gamma_{1}:=\left\{(t, \tau) \in U^{*} \times V^{*}: t^{d}-\tau^{d}=0\right\}$. Hence and from the simple fact that

$$
\mathcal{L}_{0}\left(F \mid \Gamma_{1}\right)=\max _{i=1}^{d} \mathcal{L}_{0}\left(F \mid \Gamma_{1 i}\right),
$$

where $\Gamma_{1 i}:=\left\{(t, \tau) \in U^{*} \times V^{*}: \tau=\theta^{i} t\right\}$ and $\theta$ is a primitive root of unity of degree $d$, we get

$$
\mathcal{L}_{0}(F)=\max _{i=1}^{d} \operatorname{ord}\left(\varphi\left(t^{q}\right)-\psi\left(\left(\theta^{i} t\right)^{p}\right)\right)
$$

We easily check that $\left\{\theta^{i p}: 1 \leq i \leq d\right\}=\left\{\eta^{i}: 1 \leq i \leq q\right\}$. Hence

$$
\max _{i=1}^{d} \operatorname{ord}\left(\varphi\left(t^{q}\right)-\psi\left(\left(\theta^{i} t\right)^{p}\right)\right)=\max _{i=1}^{q} \operatorname{ord}\left(\varphi\left(t^{q}\right)-\psi\left(\eta^{i} t^{p}\right)\right) .
$$

From (15), (16) and the definition of $\nu$ we get

$$
\mathcal{L}_{0}(F)=\max _{i=1}^{q} \operatorname{ord}\left(\varphi\left(t^{q}\right)-\psi\left(\eta^{i} t^{p}\right)\right)=d \nu .
$$

Hence and from (14) for $|(t, \tau)|<r$ we get

$$
|F(t, \tau)| \geq C_{2}|t|^{d \nu}
$$

Now, we estimate $\varrho(x, Y)$ from below for $x \in X \cap P(\delta)$. Since $P(2 \delta) \subset \Omega$, then there exists $y_{0} \in Y \cap P(2 \delta)$ such that $\varrho(x, Y)=\varrho\left(x, y_{0}\right)$. Let $x=\left(t^{d}, \varphi\left(t^{q}\right)\right), y_{0}=\left(\tau_{0}^{d}, \psi\left(\tau_{0}^{p}\right)\right)$. 
Since for $x \in P(\delta),|t|<\delta^{1 / d}<r$ and for $y_{0} \in P(2 \delta),\left|\tau_{0}\right|<(2 \delta)^{1 / d}<r$, then from (17) and (11) we get

$$
\varrho(x, Y)=\varrho\left(x, y_{0}\right)=\left|F\left(t, \tau_{0}\right)\right| \geq C_{2}|t|^{d \nu} \geq C|x|^{\nu},
$$

where $C:=C_{2} / D_{1}^{\nu}$.

Summing up, from (13) and (18) for $x \in X \cap P(\delta)$ we obtain

$$
C|x|^{\nu} \leq \varrho(x, Y) \leq D|x|^{\nu}
$$

which gives that $\mathcal{L}_{0}(X, Y)=\nu$.

This ends the proof.

We shall now give a second formula for $\mathcal{L}_{0}(X, Y)$ in terms of the first version of the Puiseux Theorem ([ $\left.\mathrm{E}_{1}\right]$, II.6.1) in the two-dimensional case.

First we give a simple lemma. Let $\Omega$ be a neighbourhood of $0 \in \mathbb{C}^{2}, h: \Omega \rightarrow \mathbb{C}$ a distinguished pseudopolynomial in $y$ of degree $r$ and $Z:=V(h)$. Assume additionally that $\hat{h} \in \mathcal{O}^{2}$ is irreducible and that $W \ni t \mapsto\left(t^{r}, \lambda(t)\right) \in \Omega$ is a parametrization of $Z$.

Lemma 1. If there exist a positive integer $D$, a disc $\Delta=\{t \in \mathbb{C}:|t|<\delta\}$ and functions $\gamma_{1}, \ldots, \gamma_{r}$ - holomorphic in $\Delta$, such that $\left\{t \in \mathbb{C}:|t|<\delta^{D / r}\right\} \subset W$ and $h\left(t^{D}, y\right)=\prod_{i=1}^{r}\left(y-\gamma_{i}(t)\right)$, then

(a) $r \mid D$,

(b) after an appropriate renumbering of $\gamma_{i}$ we have $\gamma_{i}(t)=\lambda\left(\varepsilon^{i} t^{D / r}\right)$ in $\Delta$ where $\varepsilon$ is a primitive root of unity of degree $r$.

Proof. Let $\Phi(t):=\left(t^{r}, \lambda(t)\right)$ and $\Psi_{i}(t):=\left(t^{D}, \gamma_{i}(t)\right)$. Put $\delta_{i}: \Delta \ni t \mapsto \Phi^{-1} \circ \Psi_{i}(t) \in W$. The function $\delta_{i}$ is continuous and $\left[\delta_{i}(t)\right]^{r}=t^{D}$ in $\Delta$. Hence it is a branch of $r$-th root of $t^{D}$ in $\Delta \backslash\{0\}$, so, it is holomorphic in $\Delta$. Hence we easily get that $r \mid D$ and there exists $j$ that $\gamma_{i}(t)=\lambda\left(\varepsilon^{j} t^{D / r}\right)$ for $t \in \Delta$. Since $h$ is an irreducible polynomial, then $\gamma_{i}$ are different. Hence by a renumbering we get $\gamma_{i}(t)=\lambda\left(\varepsilon^{i} t^{D / r}\right)$ for $t \in \Delta$. This ends the proof of the lemma.

Let us return to the announced theorem. Let $\Omega$ be a neighbourhood of $0 \in \mathbb{C}^{2}, X, Y$ - analytic curves in $\Omega$ and $X \cap Y=\{0\}$. Assume that $X=V(f), Y=V(g)$, where $f$ and $g$ are distinguished pseudopolynomials in $y$ of degree $p$ and $q$, respectively.

THEOREM 4. If there exist a positive integer $D$ and holomorphic functions $\alpha_{1}, \ldots, \alpha_{p}$, $\beta_{1}, \ldots, \beta_{q}$ in a neighbourhood of $0 \in \mathbb{C}$ such that ord $\alpha_{i} \geq D$, ord $\beta_{i} \geq D$ and

$$
\begin{aligned}
& f\left(t^{D}, y\right)=\prod_{i=1}^{p}\left(y-\alpha_{i}(t)\right), \\
& g\left(t^{D}, y\right)=\prod_{j=1}^{q}\left(y-\beta_{j}(t)\right),
\end{aligned}
$$

then

$$
\mathcal{L}_{0}(X, Y)=(1 / D) \max _{i=1}^{p} \max _{j=1}^{q} \operatorname{ord}\left(\alpha_{i}-\beta_{j}\right)
$$


Pro of. By Proposition 3 we may assume that $X, Y$ generate irreducible germs at 0 . In consequence, we may also assume that $\hat{f}, \hat{g}$ are irreducible in $\mathcal{O}^{2}$. Let now $U \ni t \mapsto$ $\left(t^{p}, \varphi(t)\right) \in X, V \ni t \mapsto\left(t^{q}, \psi(t)\right) \in Y$ be parametrizations of $X$ and $Y$. Let us take such a small $\delta$ that the functions $\alpha_{1}, \ldots, \alpha_{p}, \beta_{1}, \ldots, \beta_{q}$ are defined in $\Delta:=\{t \in \mathbb{C}:|t|<\delta\}$ and $\left\{t \in \mathbb{C}:|t|<\delta^{D / p}\right\} \subset U,\left\{t \in \mathbb{C}:|t|<\delta^{D / q}\right\} \subset V$ hold. Then from (19), by Lemma 1 , we get $p|D, q| D$ and, after a renumbering,

$$
\alpha_{i}(t)=\varphi\left(\varepsilon^{i} t^{D / p}\right), \quad \beta_{j}(t)=\psi\left(\eta^{j} t^{D / q}\right) \quad \text { for } t \in \Delta,
$$

where $\varepsilon, \eta$ are primitive roots of unity of degree $p$ and $q$, respectively. Hence we immediately obtain that ord $\varphi \geq p$, ord $\psi \geq q$ and

$$
(1 / D) \operatorname{ord}\left(\alpha_{i}-\beta_{j}\right)=(1 / p q) \operatorname{ord}\left(\varphi\left(\varepsilon^{i} t^{q}\right)-\psi\left(\eta^{j} t^{p}\right)\right) .
$$

Since for every $i \in\{1, \ldots, p\}$ the function $\left\{t \in \mathbb{C}: \varepsilon^{i} t \in U\right\} \ni t \mapsto\left(t^{p}, \varphi\left(\varepsilon^{i} t\right)\right) \in X$ is a parametrization of $X$ and $\operatorname{ord} \varphi \geq p$, ord $\psi \geq q$, then from Theorem 3 we have

$$
(1 / D) \max _{j=1}^{q}\left(\operatorname{ord}\left(\alpha_{i}-\beta_{j}\right)\right)=(1 / p q) \max _{j=1}^{q}\left(\operatorname{ord}\left(\varphi\left(\varepsilon^{i} t^{q}\right)-\psi\left(\eta^{j} t^{p}\right)\right)\right)=\mathcal{L}_{0}(X, Y) .
$$

Hence we get (20). This ends the proof.

R e m ark. The assumptions in Theorem 4 are not restrictive, because for any analytic curves $X, Y$ in $\Omega, X \cap Y=\{0\}$, there is a linear change of coordinates in $\mathbb{C}^{2}$ such that in these new coordinates $X$ and $Y$ satisfy these assumptions.

4. Concluding remarks. Let $\Omega$ be a neighbourhood of $0 \in \mathbb{C}^{m}, X, Y \subset \Omega$ analytic curves such that $X \cap Y=\{0\}$. Denote by $C(X), C(Y)$ the tangent cones at 0 to $X, Y$, respectively. From Theorem 3 we obtain

Corollary ([T], Cor. 3.4). Under the above assumptions

(a) $\mathcal{L}_{0}(X, Y) \geq 1$

(b) $\mathcal{L}_{0}(X, Y)=1$ if and only if $C(X) \cap C(Y)=\{0\}$.

Proof. Let $H_{1}:=\left\{\left(z_{1}, \ldots, z_{m}\right) \in \mathbb{C}^{m}: z_{1}=0\right\}$. Without loss of generality, at the cost of linear change of coordinates, we may assume that $H_{1} \cap C(X)=\{0\}$, $H_{1} \cap C(Y)=\{0\}$. We may also assume (see Proposition 3 ) that $X$ and $Y$ generate irreducible germs at $0 \in \mathbb{C}^{m}$. Then $X, Y$ satisfy the assumptions of Theorem 3 and hence (a) is obvious. Moreover, $\mathcal{L}_{0}(X, Y)=1$ if and only if $\operatorname{ord}\left(\varphi\left(t^{q}\right)-\psi\left(t^{p}\right)\right)=p q$. But this holds if and only if $X$ and $Y$ have different tangent lines at 0 .

Let $X, Y$ be as at the beginning of this section. Let $\mu(X, Y)$ mean the intersection multiplicity of $X$ and $Y$ at 0 and $\operatorname{deg} X, \operatorname{deg} Y$ degrees of $X$ and $Y$ at 0. P. Tworzewski [T] proved that

$$
\mathcal{L}_{0}(X, Y) \leq \mu(X, Y)-\operatorname{deg} X \operatorname{deg} Y+1 .
$$

Now we give an example for which the equality in (21) does not hold.

ExAmple. Let $X=\left\{(x, y, z) \in \mathbb{C}^{3}: x^{3}-y z=0, y^{2}-x z=0, z^{2}-x^{2} y=0\right\}$, $Y=\left\{(x, y, z) \in \mathbb{C}^{3}: x^{3}-\varepsilon y z=0, y^{2}-\varepsilon x z=0, z^{2}-\varepsilon x^{2} y=0\right\}$, where $\varepsilon$ is a primitive root of unity of degree 3 . It is easy to show ([M], Ex. 3.2) that $X$ and $Y$ generate irreducible germs at $0 \in \mathbb{C}^{3}$. Moreover, $\mathbb{C} \ni t \mapsto\left(t^{3}, t^{4}, t^{5}\right) \in X, \mathbb{C} \ni t \mapsto\left(t^{3}, t^{4}, \varepsilon^{2} t^{5}\right) \in Y$ 
are their parametrizations. Obviously, $\operatorname{deg} X=3, \operatorname{deg} Y=3$ and $\mu(X, Y)=13$ (it can be calculated directly from the definition of the multiplicity, given in $[\mathrm{T}]$ ). Whereas, from Theorem 3 we have

$$
\begin{aligned}
\mathcal{L}_{0}(X, Y) & =(1 / 9) \max _{i=1}^{3} \min \left(\operatorname{ord}\left(t^{12}-\varepsilon^{i} t^{12}\right), \operatorname{ord}\left(t^{15}-\varepsilon^{2+2 i} t^{15}\right)\right) \\
& =(1 / 9) \max (12,12,15)=(5 / 3) .
\end{aligned}
$$

Acknowledgements. We thank S. Spodzieja for helpful discussions and a technical help in preparing the paper for publication and P. Tworzewski for discussions on the intersection multiplicity.

\section{References}

[CK] J. Chạdzyński, T. Krasiński, A set on which the local Eojasiewicz exponent is attained, Ann. Polon. Math. 67 (1997), 191-197.

[CT] E. Cygan, P. Tworzewski, Proper intersection multiplicity and regular separation of analytic sets, Ann. Polon. Math. 59 (1994), 293-298.

[ $\left.\mathrm{E}_{1}\right] \quad \mathrm{S}$. Łojasiewicz, Introduction to Complex Analytic Geometry, Birkhäuser, Basel, 1991.

$\left[\mathrm{E}_{2}\right]$ S. Łojasiewicz, Ensembles semi-analytiques, Inst. de Hautes Études Scientifiques, Bures-sur-Yvette, 1965.

[M] A. Miodek, On some method of calculating a multiplicity, Bull. Soc. Sci. Lett. Łódź 37 (1987), No. 7, 9 pp.

[T] P. Tworzewski, Isolated intersection multiplicity and regular separation of analytic sets, Ann. Polon. Math. 58 (1993), 213-219. 